Mal J Nutr 27(3): 483-494, 2021

\title{
Improvement in body composition of adult post- bariatric surgery patients at one-year follow-up at a public hospital in United Arab Emirates
}

\section{Amita Attlee $^{1^{*}}$, Hayder Hasan ${ }^{2,3^{* *}}$, Latefa Mohammed Rashed ${ }^{4}$, Muna Al-Haway \& Abdul-Wahid Al-Wahedi ${ }^{5}$}

${ }^{1}$ Department of Nutrition and Health, College of Medical and Health Sciences, United Arab Emirates University, Al Ain, United Arab Emirates; ${ }^{2}$ Department of Clinical Nutrition and Dietetics, College of Health Sciences, University of Sharjah, UAE; ${ }^{3}$ Diabetes and Metabolic Syndrome Research Group, Sharjah Institute for Medical Research, University of Sharjah, Sharjah, United Arab Emirates; ${ }^{4}$ Nutrition Section, Ministry of Health and Prevention, Dubai, United Arab Emirates; ${ }^{5}$ Al Qassimi Hospital, Sharjah, Ministry of Health and Prevention, United Arab Emirates

\begin{abstract}
Background: Data on postoperative follow-ups and bariatric surgery (BS) outcomes performed in the United Arab Emirates (UAE) are crucial for registry. This study assessed the one-year postoperative changes in body composition and metabolic profiles in BS patients. Methods: The medical and dietetics records of 51 adult patients who underwent BS in the largest public hospital in Sharjah, UAE were reviewed. Data on body weight and composition, as well as metabolic profile (blood glucose and lipid levels) from the initial until the last hospital visit were recorded. Results: The median (interquartile range) follow-up period was 6.0 (8.0) months. The patients had significant total weight loss [19.3 (12.6)\%] and reduction in body mass index (BMI) [18.0 (13.6)\%] of approximately 7 BMI points. Moreover, the patients' body composition improved significantly; loss was the highest in fat mass [-30.9 (22.1)\%] and the least in lean body mass [-8.6 (8.4)\%]. There was a steady decline in all body composition variables with a longer duration of follow-up visits from $1-3$ months to $10-12$ months. The fat mass $(-40.3 \%)$ :lean body mass $(-10.7 \%)$ loss ratio was 3.8:1 at 10-12 months. The patients' metabolic status was normal during the last postoperative visit. Conclusions: Incremental improvements in body composition of patients were evident with longer follow-up visits up to one year after BS. Hence, patients should attend regular follow-up visits after BS. Moreover, accurate and complete documentations of medical and dietetics visits are mandated.
\end{abstract}

Keywords: bariatric surgery; body composition; postoperative

Corresponding author: *Amita Attlee, Department of Nutrition and Health,

College of Medicine and Health Sciences, University Arab Emirates University, PO Box 15551, Al Ain, United Arab Emirates

Tel: +971-3-713-4571; E-mail: amita.attlee@uaeu.ac.ae

**Hayder Hasan, Department of Clinical Nutrition and Dietetics,

College of Health Sciences, University of Sharjah, PO Box 27272, Sharjah, United Arab Emirates

Tel.: +971-6-505-7512; E-mail: haidarah@sharjah.ac.ae

doi: https://doi.org/10.31246/mjn-2021-0024 


\section{INTRODUCTION}

The prevalence rates of overweight and obesity $(43.0 \%$ and $32.3 \%$, respectively) are high in the United Arab Emirates (UAE) (Sulaiman et al., 2017). Recently, the risk of metabolic complications according to waist circumference and waist-to-hip ratio $(52.4 \%$ and $56.5 \%$, respectively) have substantially increased in the UAE (Sulaiman et al., 2017). Any type of bariatric surgery (BS) is considered a frontline treatment for severe obesity, thereby leading to superior weight loss and maintenance along with improvements in medical morbidities, compared with nonsurgical interventions (Pareek et al., 2018; Ruban et al., 2019). A previous report showed that the number of BS performed worldwide from 2011 to 2013 had significantly increased (98.0\%) (Angrisani et al., 2015). Globally, the most common procedure is sleeve gastrectomy (SG) $(58.6 \%)$, followed by Roux-en-Y gastric bypass (RYGB) at $31.2 \%$ (Ramos et al., 2019).

Studies have shown improvements in body composition after BS (Andersson et al., 2019; Maïmoun et al., 2019). Specifically, significant improvement in glycemic control and reduced 10-year cardiovascular risk were reported at 12-24 months after RYGB (Andersson et al., 2019; Pareek et al., 2018). The mechanisms seem to extend beyond the magnitude of weight loss alone and include improvements in incretin profiles, insulin secretion, and insulin sensitivity, which all lead to improved patient outcomes and reduced cardiovascular risk factors (Prateek et al., 2018).

The UAE is a member of the BS group/society under the International Federation for the Surgery of Obesity and Metabolic Disorders. There were 2,843 BS procedures conducted in the UAE from 2015 to 2018; the most common being SG (84.0\%), followed by RYGB (13.0\%), adjustable gastric banding (AGB) in 3.0\%, and mini gastric bypass (MGB) in 3.0\% (Ramos et al., 2019). Although thousands of BS are performed annually in the UAE, data in the national registry of BS cases are incomplete (Ramos et al., 2019). Moreover, information on the outcomes of $\mathrm{BS}$ in patients in the UAE is limited.

To the best of our knowledge, only one study was conducted at a single BS centre in the UAE. This research focused on the outcomes (weight and metabolic profiles) of BS after one year (Abusnana et al., 2015). Such information is important not only for healthcare providers, but also for patients, as it can help build confidence in BS and its health benefits. There was a substantial decrease in the patient follow-up rate $(39.0 \%)$ at the end of one year after BS in the UAE study (Abusnana et al., 2015).

Hence, the current study aimed to assess the postoperative changes in body composition and metabolic profiles in BS patients during their last visit one year after the surgery in one of the largest public hospitals in UAE.

\section{METHODS}

We retrospectively assessed the medical and dietetics records of patients in the largest public hospital in Sharjah under the Ministry of Health and Prevention (MOHAP), UAE. This hospital is among the 19 BS centres in the country (Ramos et al., 2019) and adopts the international standards for the eligibility criteria for BS set by the National Institutes of Health and American Society for Metabolic and Bariatric Surgery (MOHAP, 2019).

SG and MGB were the most common BS procedures carried out. The surgical procedures (Karamanakos et al., 2008; Lee et al., 2012) were conducted according to the BS Management Guidelines published under the Clinical 
Practice Guidelines by the MOHAPUAE (MOHAP, 2019). SG was performed laparoscopically, and it involved the dissection of the stomach with a linear stapler starting $3 \mathrm{~cm}$ from the pylorus up to the angle of His. A $60-80-\mathrm{mL}$ gastric sleeve tube was placed, and $85 \%$ of the stomach was excised. MGB was performed by creating a long-sleeved gastric tube that is approximately 2.0$\mathrm{cm}$ wide along the lesser curvature starting below the incisurae angularis to the angle of His. Then, antecolic Billroth II-type loop gastroenterostomy was conducted in the small intestine $200 \mathrm{~cm}$ distal to the ligament of Treitz. Patients could choose the type of procedure based on their medical condition and the surgeon's preference.

The medical and dietetics records of all male and female adult patients aged 18 years and above who underwent BS between January 1, 2014 and April 30, 2014 were reviewed in April 2015. Electronic and paper records of all medical and dietetics data from the initial visit (time of surgery) until the last visit (April 30, 2015) were reviewed. At least one post-BS follow-up visit was considered for inclusion. On the contrary, patient records without any follow-up visits after BS were excluded. Accordingly, the medical and dietetics records of 66 patients who underwent BS during the above-mentioned period were reviewed. However, due to the lack of follow-up visits/data, only 51 patients were included in the analysis. Figure 1 shows the recruitment process and follow-up visits of patients after BS.

To elaborate, patients' records were reviewed to collect the following information: demographic characteristics, including age, sex, marital status, and nationality; medical records of the date and type of BS, blood pressure, and metabolic profile based on the biochemical tests [lipid profile (total cholesterol, TC; triglyceride, TG; low density lipoprotein, LDL; and high density lipoprotein, HDL), blood glucose, and glycosylated haemoglobin (HbA1c) levels]; and dietetics records on physical measurements, including body weight, height, body mass index (BMI), body composition in terms of fat mass (FM), body fat percentage $(\% \mathrm{BF})$, lean body mass (LBM), total body water (TBW), and waist-to-hip ratio (WHR). Body weight and height measurements were collected using the appropriate equipment (SECA, Model 286, Germany) and BMI was calculated accordingly using the information on body weight and height. Body composition analysis (BCA) was conducted via bioelectrical impedance using the eight-electrode method (SECA, Model mBCA514, Germany). WHR was automatically calculated on the BCA machine (ACCUNIQ BC 360). The comorbidity data of patients were not included.

Data analysis was performed using the Statistical Package for the Social Sciences software version 24.0 (IBM SPSS, Chicago, IL, the USA). Non-parametric tests were used after assessing the normality distribution of data using the Shapiro-Wilk test. Descriptive statistics were presented as frequencies and percentages, medians, and interquartile range (IQR). The Wilcoxon signed-rank test was used to compare the changes in body composition variables between the initial (preoperative period) and the last follow-up visits. All data were tested at a $5 \%$ significance level $(p<0.05)$.

This study was conducted in accordance with the Code of Ethics of the World Medical Association (Declaration of Helsinki). The research protocol was approved by the Research Ethics Committee of the Al Qassimi Clinical Research Centre, Ministry of Health and Prevention, Sharjah, United Arab Emirates (UG004/2015-02-19) that 


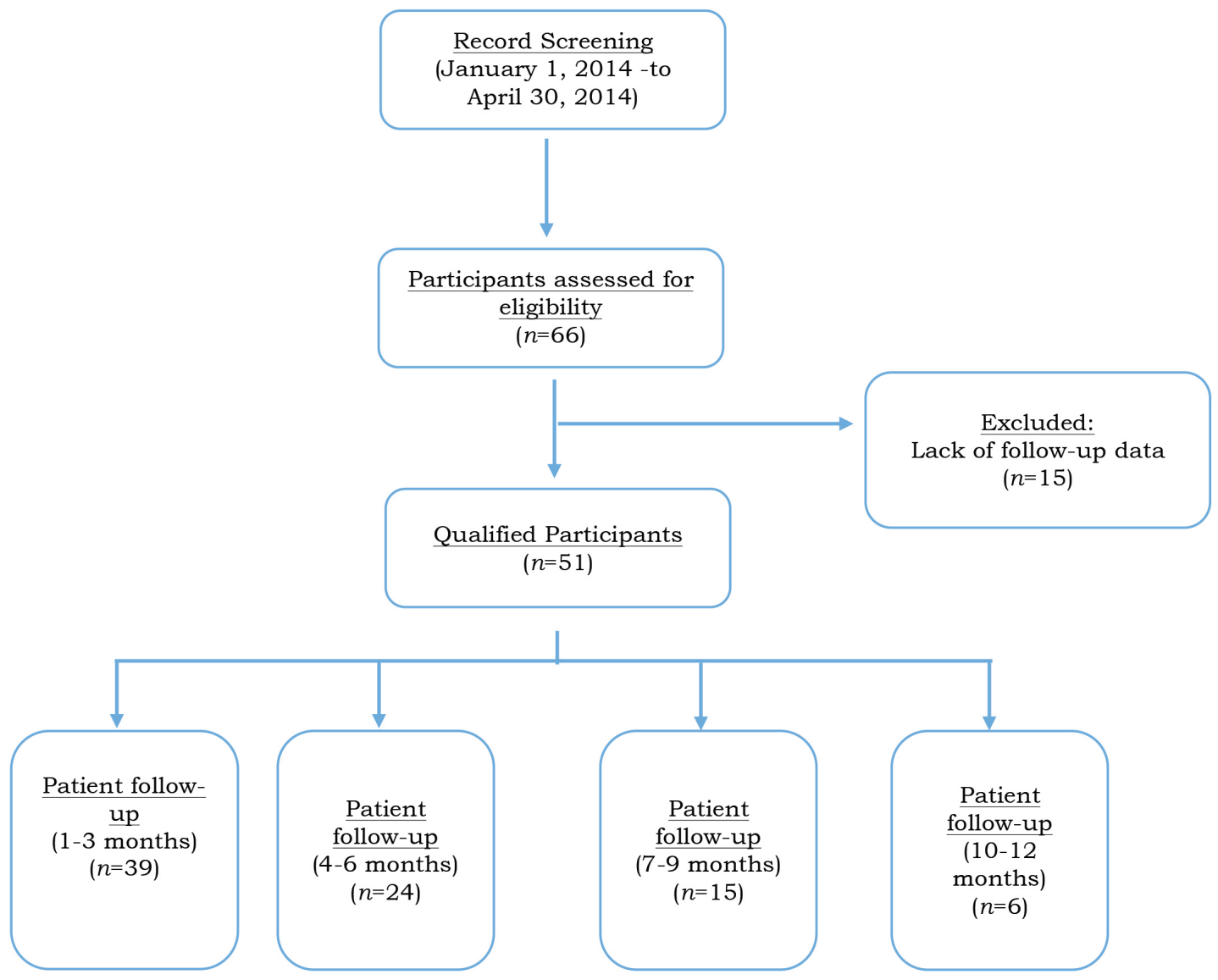

Figure 1. Flowchart of patient recruitment process and follow-up after bariatric surgery

considered patients' consent for their data to be used in future retrospective studies.

\section{RESULTS}

The demographic characteristics of BS patients $(N=51)$ are summarised in Table 1 . There were $47(92.2 \%)$ women and $4(7.8 \%)$ men; 38 of 51 patients were married and 13 were not. Most patients were UAE nationals (Emiratis; $n=45,88.2 \%)$, while six $(11.8 \%)$ were expatriates from other Arab and Southeast Asian countries. The median (IQR) age of the patients was 32.0 (10.0) years. In total, 38 underwent SG and 13 MGB surgery.
The median (IQR) follow-up period was 6.0 (8.0) months. Moreover, the average number of postoperative patient visits for medical consultation was 4.8 (maximum of 16), and the average number of dietary counselling visits during the follow-up period was 3.5 (up to 8 visits). None of the patients visited the hospital beyond one year after BS. Based on the records, their visits were limited within the first few months after BS.

Table 2 shows the changes in the physical measurements and body composition measures of patients during the postoperative follow-up period. The average weight of the patients was 98.9 
Table 1. Demographic characteristics of the participants $(N=51)$ and type of surgery

\begin{tabular}{|c|c|c|}
\hline Characteristics of the participants & Median (IQR) & $\begin{array}{c}\text { Patients } \\
n(\%)\end{array}$ \\
\hline \multicolumn{3}{|l|}{ Sex } \\
\hline Female & & $47(92.2)$ \\
\hline Male & & $4(7.8)$ \\
\hline Marital status & & $38(74.5)$ \\
\hline Married & & $13(25.5)$ \\
\hline Single & & \\
\hline \multicolumn{3}{|l|}{ Nationality } \\
\hline UAE nationals (Emiratis) & & $45(88.2)$ \\
\hline Expatriates & & $6(11.8)$ \\
\hline Age (years) & $32.0(10.0)$ & \\
\hline \multicolumn{3}{|l|}{ Type of bariatric surgery } \\
\hline Sleeve Gastrectomy & & $38(74.5)$ \\
\hline Mini Gastric Bypass & & $13(25.5)$ \\
\hline Follow-up period after BS (months) & $6.0(8.0)$ & \\
\hline
\end{tabular}

(23.4) $\mathrm{kg}$ at the time of surgery and 81.1 (15.1) kg during the last follow-up visit. Total weight loss [19.3 (12.6)\%] during the postoperative period was significant $(p<0.001)$.

The preoperative median (IQR) BMI of all patients was $39.5(7.1) \mathrm{kg} / \mathrm{m}^{2}$, which indicated borderline morbid obesity. However, it decreased to 32.8 (5.6) kg/ $\mathrm{m}^{2}$; hence, the patients were categorised under grade I obesity during their last visit. The value reduced by $18.0 \%$ (almost 7 BMI points), which was highly significant $(p<0.001)$.
During the postoperative period, body FM significantly reduced [30.9 (22.1)\%] $(p<0.001)$. Moreover, FM decreased from $44.9(18.1) \mathrm{kg}$ in the initial visit to 31.1 (9.0) $\mathrm{kg}$ during the last follow-up visit. Patients' \%BF reduced from 43.9 (7.1)\% at the time of surgery to 36.1 (6.7)\% on the last follow-up visit. Concurrently, there was a significant decrease in LBM from 56.7 (9.7) $\mathrm{kg}$ to 52.4 (8.2) $\mathrm{kg}$ in the postoperative period. In addition, TBW significantly decreased from 41.0 (7.3) $\mathrm{kg}$ to 37.6 (6.0) $\mathrm{kg}$. Conversely, there was a significant reduction in WHR from

Table 2. Medians and interquartile ranges (IQRs) for changes in body composition after bariatric surgery

\begin{tabular}{lcccc}
\hline \multirow{2}{*}{ Variables } & \multicolumn{3}{c}{ Median (IQR) } & \multirow{2}{*}{-value } \\
\cline { 2 - 4 } & Initial visit & Last visit & Difference (\%) & $<0.001$ \\
Weight $(\mathrm{kg})$ & $98.9(23.4)$ & $81.1(15.1)$ & $-19.3(12.6)$ & $<0.001$ \\
BMI $\left(\mathrm{kg} / \mathrm{m}^{2}\right)$ & $39.5(7.1)$ & $32.8(5.6)$ & $-18.0(13.6)$ & $<0.001$ \\
FM $(\mathrm{kg})$ & $44.9(18.1)$ & $31.1(9.0)$ & $-30.9(22.1)$ & $<0.001$ \\
BF $(\%)$ & $43.9(7.1)$ & $36.1(6.7)$ & $-18.3(15.4)$ & $<0.001$ \\
LBM $(\mathrm{kg})$ & $56.7(9.7)$ & $52.4(8.2)$ & $-8.6(8.4)$ & $<0.001$ \\
TBW $(\mathrm{kg})$ & $41.0(7.3)$ & $37.6(6.0)$ & $-8.9(7.8)$ & $<0.001$ \\
WHR & $1.0(0.1)$ & $0.9(0.1)$ & $-9.1(6.8)$ & \\
\hline
\end{tabular}

BMI, body mass index; FM, fat mass; \%BF, body fat percentage; LBM, lean body mass; TBW, total body water; and WHR, waist-to-hip ratio 
$0.96(0.07)$ at the time of surgery to 0.90 (0.10) during the last visit.

Figure 2 shows the postoperative changes in the body composition measures of patients, which were expressed as percentage, during the follow-up visits. There was a constant decline in all body composition measures from $1-3$ to $10-12$ months. Moreover, there was a linear reduction in $\mathrm{FM}$ by $40.3 \%$ in patients who visited the hospital up to one year. Meanwhile, there was only a $21.2 \%$ reduction in FM within 1-3 months after BS during the follow-up period. LBM consistently decreased $(2.4 \%$ at $1-3$ months after BS vs. $10.7 \%$ at $10-12$ months after $B S$ ). The FM:LBM loss ratio at 10-12 months was 3.8:1. Similar trends of amplified improvements in other body composition measures were observed in patients who had postoperative follow-up until 10-12 months.
The systolic and diastolic blood pressures of the patients were normal from the time of surgery until the last follow-up visit. The average haemoglobin level significantly improved from 10.4 (2.5) $\mathrm{g} / \mathrm{dL}$ to $11.8(1.92) \mathrm{g} / \mathrm{dL}(p<0.05)$ one year after BS.

Only biochemical measurements available during the last visit were considered. The averages of these selected biochemical variables showed that the metabolic profiles of patients were within the normal ranges (Table 3). However, LDL cholesterol level was $2.86(1.0) \mathrm{mmol} / \mathrm{L}$ at the end of the follow-up visit, which was still marginally above the normal range $(<2.5$ $\mathrm{mmol} / \mathrm{L}$ ). Moreover, total cholesterol level was within the normal range [4.4 (1.2) $\mathrm{mmol} / \mathrm{L}$; reference value: $<5.2$ $\mathrm{mmol} / \mathrm{L}]$. Triglyceride level was 0.9 (0.4) $\mathrm{mmol} / \mathrm{L}$, which was lower than the normal reference value $(<1.7 \mathrm{mmol} / \mathrm{L})$,

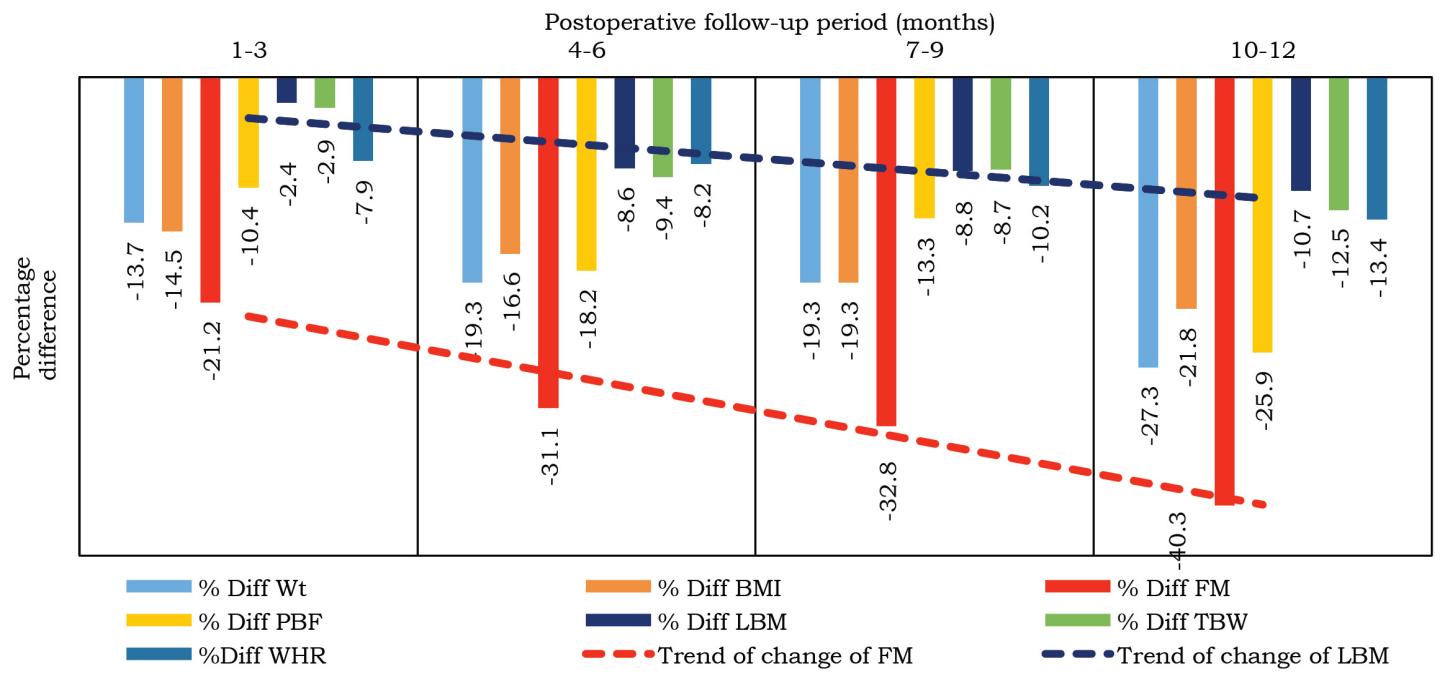

\%Diff Wt, percent difference in weight; \%Diff BMI, percent difference in body mass index; $\%$ Diff FM, percent difference in fat mass; \%Diff PBF, percent difference in percent body fat; \%Diff LBM, percent difference in lean body mass; \% Diff TBW, percent difference in total body water; \%Diff WHR, percent difference in waist-to-hip ratio

Follow-up period after BS: $1-3$ months $(n=39)$; 4-6 months $(n=24)$; $7-9$ months $(n=15)$; 10-12 months $(n=6)$

Figure 2. Body composition of patients at different follow-up periods after bariatric surgery 
Table 3. Medians and interquartile ranges (IQRs) of metabolic profiles at the last follow-up visit after bariatric surgery

\begin{tabular}{lll}
\hline Variables & Median (IQR) & Reference value \\
\hline Total cholesterol level (mmol/L) & $4.4(1.2)$ & $<5.2$ \\
Triglyceride level (mmol/L) & $0.9(0.4)$ & $<1.7$ \\
LDL level (mmol/L) & $2.9(1.0)$ & $<2.6$ \\
HDL level $(\mathrm{mmol} / \mathrm{L})$ & $1.4(0.2)$ & Men: $1.0-1.3$ \\
FBG level $(\mathrm{mmol} / \mathrm{L})$ & $5.4(0.6)$ & Women: $1.3-1.50$ \\
HbA1c $(\%)$ & $5.7(0.4)$ & $<5.6$ \\
\hline
\end{tabular}

LDL, low density lipoprotein; HDL, high density lipoprotein; FBG, fasting blood glucose; HbA1c, glycosylated haemoglobin

while HDL cholesterol level was 1.3 (0.2) $\mathrm{mmol} / \mathrm{L}$, which was at a desirable level (normal reference values: 1.0-1.3 $\mathrm{mmol} / \mathrm{L}$ in men and $1.3-1.5 \mathrm{mmol} / \mathrm{L}$ in women). Similarly, the patients' blood glucose level was within normal range after surgery. Fasting blood glucose level and $\mathrm{HbA} 1 \mathrm{c}$ were 5.4 (0.6) $\mathrm{mmol} / \mathrm{L}$ and $5.7(0.4) \%$, respectively, thus within the normal values $(<5.6 \mathrm{mmol} / \mathrm{L}$ and $<6.0 \%$, respectively).

\section{DISCUSSION}

In the Eastern Mediterranean region, the UAE is one of the countries with the highest proportion of overweight and obese adults (World Health Organization / Regional Office for the Eastern Mediterranean, 2018). The incidences of obesity, type II diabetes mellitus, and metabolic syndrome among young adults in the UAE have been a cause of concern (Alzaabi et al., 2019). Concurrently, the number of individuals who underwent BS has significantly increased in the country based on global statistics (DHA, 2019; Ramos et al., 2019). According to the demographic characteristics of our population, younger individuals (aged 32 years) and women $(92.2 \%)$ were more likely to undergo BS; this was similar to the trend reported in earlier studies in the UAE (40 years and $65.1 \%$, respectively) (Alia et al., 2019) and other countries worldwide (39.0 years and $72 \%$, respectively) (Luca et al., 2021).

This retrospective study focused on the one-year postoperative changes in body composition and metabolic profiles of BS patients in 1 of the 19 registered clinical sites for BS in the UAE. Previous studies with short-term follow-up until 12 months have shown substantial weight loss and improvements in comorbidities after BS procedures, such as RYGB, SG, and AGB (Andersson et al., 2019; Schwoerer et al., 2017). The current study revealed that about $74.5 \%$ and $25.5 \%$ of patients underwent SG and MGB. SG is the most commonly performed BS procedure in countries worldwide and in the Middle East region (Nimeri et al., 2017; Ramos et al., 2019).

In the current study, the average post-BS follow-up period was 6.0 months. None of these patients visited the hospital for clinical consultations beyond one year after BS. In fact, consultations with healthcare professionals after BS were insufficient; the average number of physician visits was 4.8 , and the average dietitian visits during the follow-up period was only 3.5. Data in literature have shown that higher adherence to physician visits after BS was associated with better weight loss outcomes (Lujan et al., 2020). In one report, although there was 
a need for postoperative care, only 34\% patients completed their recommended follow-up visits in person, and $66 \%$ were lost to follow-up before one year post-BS (Lujan et al., 2020). In another study, only $44.7 \%$ committed to follow-ups during the first five years after BS (Luca et al., 2021). The actual reason for such low retention is unknown. Perhaps, the patients who have undergone BS did not feel the need for further intervention after surgery $(29.5 \%)$ or they believed that behavioural interventions have minimal effects because these might not have resulted in long-term weight loss prior to surgery (Luca et al., 2021).

Patients lost an average of almost one-fifth $(19.3 \%)$ of their initial body weight and one-third of body FM (30.9\%) during the follow-up period. Similar to the findings of a previous study on individuals in the UAE (Abusnana et al., 2015), the reduction in FM was most remarkable after BS. However, the patients in the current study had a lower reduction in body FM than those in the previous study (30.9\% vs. 35\%). Moreover, compared to an earlier study (Abusnana et al., 2015), the current study had lower reductions in LBM $(8.6 \%$ vs. $26.8 \%)$ and $\mathrm{TBW}(8.9 \%$ vs. $32 \%)$. The percentage of muscle mass loss one year after SG was reportedly up to 22\% (Palacio et al., 2019). Our results showed that the decline in body composition measures increased with the duration of follow-up period. That is, the lowest decline was observed within 1-3 months and the highest within 10-12 months after BS. Furthermore, $\mathrm{FM}$ reduction reached up to $40.3 \%$ in patients who attended the follow-up sessions up to 10-12 months, but those who attended the follow-up sessions within 1-3 months after BS only reached a reduction of up to $21.2 \%$ in FM.

There is a lack of consensus regarding the loss of fat-free mass (FFM) that is considered excessive in terms of undesirable metabolic outcomes (Nuijten et al., 2020); following which they proposed around $25 \%$ of total weight loss as the cut-off point for excessive FFM loss. Overall, FM loss has been reported to exceed lean mass loss by threefold in a cohort of adults (aged $45 \pm 12$ years) at 12 months of follow-up (FM: $-49 \% \pm 12 \%$ vs. FFM: $-14 \% \pm 6 \% ; p<0.05$ compared with the baseline values for both) (Alba et al., 2019). In the current study, the ratio of FM:LBM loss was 3.8:1 at 10-12 months after BS. In a previous study, patients who had the highest weight loss had accelerated losses of both LBM and FM with incremental LBM loss, with lean-to-fat loss ratios of 1:4.0, 1:3.6, and 1:3.0 among the respective tertiles based on the reduction in BMI per month (Zalesin et al., 2010). Nonetheless, aggressive nutritional and behavioural interventions are required to preserve lean mass after BS.

There was an improvement in central obesity among patients as reflected by the significant reduction in WHR from 0.96 to 0.90 , thereby reaching a $13.4 \%$ loss within 10-12 months after BS. In a prospective study on Hispanic adults, the presence of abdominal obesity was highlighted as predictive of mortality risk; therefore, loss of central obesity indicated a reduced risk (Gnatiuc, 2020).

Data from the literature showed that weight loss of about $25 \%$, along with improvements of comorbidities, indicated surgical success (Masnyj, Shea \& Khaitan, 2020; Tu et al., 2021). In a recent study, the success criteria called for the consideration of clear goals regarding health improvement, which include the metabolic aspects of the patients. Therefore, moving beyond a weight-centric approach to define the success and the cost-benefit of BS is recommended (Unamuno, Portincasa $\&$ Frühbeck, 2019). In a sample of 80 Emirati adults with diabetes, weight loss was within the recommended success 
rate postoperatively $(32 \%$ after two years of follow-up); however, patients also experienced significant drops in $\mathrm{HbA} 1 \mathrm{c}$, systolic and diastolic blood pressures, and LDL cholesterol and total cholesterol $(p<0.001$ for all metabolic markers) (Alnageeb et al., 2018).

The lipid profiles of the patients were within normal ranges, except for LDL, which was still marginally higher [2.86 (0.96) $\mathrm{mmol} / \mathrm{L}]$ than the normal limit during the last follow-up visit. Our findings on better lipid profiles one year after BS were supported by a previous study conducted in the UAE. Patients in the current study also showed improved glucose control (FBG: 5.40 mmol/L, HbA1c: $5.74 \%$ ), similar to the patients in another study (FBG level: 5.0 mmol/L and HbA1c: 5.3\%) (Andersson et al., 2019). Although the comorbidity data from the medical records of patients were not included in the study, improvements in their lipid profile after surgery can reduce cardiovascular risk. Indeed, such improvements have been found to significantly reduce the 10year cardiovascular risk, according to the Framingham risk equation, during the 12-24 months follow-up after RYGB surgery (Andersson et al., 2019).

To the best of our knowledge, this was one of few studies in the UAE that showed steady improvements in body composition and metabolic profile one year after BS. However, the current study had certain limitations. That is, the study used a retrospective design, and it was conducted at a single public health facility, thereby leading to restrictions in patient recruitment and inclusion of a small sample size. Moreover, the postoperative follow-up visits were not sufficient. Thus, only patients who had a minimum of one year of followup record after BS were included. Missing/incomplete patient data might be attributed to the transition phase of patient records/follow-ups from manual documentation to the use of an electronic system in the hospital during the survey period. The low return rate might have been caused by the fact that patients who had a successful weight loss did not feel the need to visit the hospital or felt the burden of additional cost for followup sessions. Furthermore, our study was restricted to one hospital, which was a referral clinical site. Thus, patients could have returned to their respective primary health care facilities for subsequent postoperative follow-ups in the absence of any complications, thereby adding to the missing data in such cases. Lastly, the small sample size limited in-depth data analysis of the follow-up visits, and comparison of outcomes one year after BS according to the types of bariatric procedures (MGB and SG) conducted in the specific hospital. Nevertheless, MGB, which is similar to RYGB, acts via the principle of restriction and malabsorption. However, MGB was reported to be superior to RYGB as it is a simpler technique and it had greater efficacy, reversibility, and revisability (Rutledge et al., 2017). Similarly, SG has emerged as a standalone procedure that can achieve weight reduction and remission of comorbidities (Mittal et al., 2021).

In the UAE, clinics conduct thousands of BS annually. However, the incomplete data in the national registry of BS cases in the country (Ramos et al., 2019) underscored the significant impact of these interventions. Therefore, documentation among health facilities should be completed and updated to reflect the outcomes of bariatric surgeries in the UAE in the context of global scenario.

\section{CONCLUSION}

The findings of this study were based on the medical and dietary records of BS patients during their last follow-up visits. 
This might have camouflaged the actual effect of BS because not all patients had regular follow-up visits until one year after surgery. Nonetheless, body weight and body composition parameters were significantly reduced after surgery. In addition, almost all biochemical markers depicted improved metabolic profiles as represented by values within the normal ranges. The importance of preoperative education inclusive of strict adherence to postoperative follow-up protocols and complete documentation cannot be overemphasised. Hence, future studies should be conducted to evaluate bariatric procedures that are most effective in improving body composition and metabolic outcomes among patients undergoing $\mathrm{BS}$ in hospitals in the UAE according to global statistics.

\section{Acknowledgments}

We acknowledge Saja Jamal, Aamina Taskeen, Asma Atef, Ekhlas Ali Derhem, and Hadeel AbdelRahman from the Department of Clinical Nutrition and Dietetics, University of Sharjah for their contributions to the implementation of this study.

\section{Authors' contributions}

AA, principal investigator, led the conceptualisation of the study, data collection, investigation, methodology, project administration, resources, supervision, validation, visualisation, writing (original draft, review and editing); $\mathrm{HH}$, led the conceptualisation of the study, data collection, formal analysis, investigation, methodology, project administration, resources, software, supervision, validation, visualisation, writing (original draft, review and editing); LMR, led the data collection, resources, writing-review and editing; MAH, led the data collection, resources, supervision, writing-review and editing; AWAW, led the data collection, resources, supervision, visualisation, writing-review and editing. All authors have critically reviewed and approved the final draft and are responsible for the content and similarity index of the manuscript.

\section{Conflict of interest}

Each author declares that he or she has no commercial associations (e.g., consultancies, stock ownership, equity interest, patent/licensing arrangement, etc.) that might pose a conflict of interest in connection with the submitted article.

\section{References}

Abusnana S, Abdi S, Tagure B, Elbagir M \& Maleckas A (2015). Bariatric surgery outcomes: a single-center study in the United Arab Emirates. Diabetes Metab Syndr Obes 8:461471. doi:10.2147/DMSO.S87861

Alba DL, Wu L, Cawthon PM, Mulligan K, Lang T, Patel S, King NJ, Carter JT, Rogers SJ, Posselt AM, Stewart L, Shoback DM \& Schafer AL (2019). Changes in lean mass, absolute and relative muscle strength, and physical performance after gastric bypass surgery. J Clin Endocrinol Metab 104(3):711-720. https://doi. org/10.1210/jc.2018-00952

Alia S, Ali HI, Zoubeidi T \& Ahmed M (2019). Nutrition knowledge and satisfaction levels of postbariatric surgery adults in the United Arab Emirates: A pilot study. $J$ Nutr Metab 2019:9148459. https://doi. org/10.1155/2019/9148459

Alnageeb H, Abdelgadir E, Khalifa A, Suliman M, Gautam S, Layani L, Subramaniam S \& Bashier A (2018). Efficacy of bariatric surgery in improving metabolic outcomes in patients with diabetes. A 24-month follow-up study from a single center in the UAE. Diabetes Metab Syndr Obes 11:459-467. https://doi.org/10.2147/ DMSO.S176761

Alzaabi A, Al-Kaabi J, Al-Maskari F, Farhood AF \& Ahmed LA (2019). Prevalence of diabetes and cardio-metabolic risk factors in young men in the United Arab Emirates: A cross-sectional national survey. Endocrinol Diabetes Metab 2(4). https://doi.org/10.1002/edm2.81

Andersson DP, Dahlman I \& Eriksson Hogling D (2019). Improved metabolism and body composition beyond normal levels following gastric bypass surgery: A longitudinal study. $J$ Intern Med 285(1):92-101.

Angrisani L, Santonicola A, Iovino P, Formisano G, Buchwald H \& Scopinaro N (2015). Bariatric surgery worldwide 2013. Obes Surg 25(10):1822-1832. https://doi.org/10.1007/ s11695-015-1657-Z

DHA (2019). Around 1,400 successful bariatric surgeries conducted in Dubai hospital in the last four years. http://www.dha.gov.ae:80/ en / DHANews / Pages / DHANews68694655018-08-2019.aspx [Retrieved July 9 2021].

Gnatiuc L (2020). Adiposity and cause-specific mortality in the Mexico City Prospective Study [Ph.D., University of Oxford]. http://ora.ox.ac. uk/objects / uuid:daa9ef30-60bc-408d-a860fb371b2cf9ea [Retrieved July 9 2021]. 
Karamanakos SN, Vagenas K, Kalfarentzos F \& Alexandrides TK (2008). Weight loss, appetite suppression, and changes in fasting and postprandial ghrelin and peptide-yy levels after roux-en-y gastric bypass and sleeve gastrectomy a prospective, double blind study. Ann Surg 247(3):401-407. https://doi. org/10.1097/SLA.0b013e318156f012

Lee WJ, Ser KH, Lee YC, Tsou JJ, Chen SC \& Chen JC (2012). Laparoscopic roux-en-Y Vs. Minigastric bypass for the treatment of morbid obesity: A 10-year experience. Obes Surg 22(12):1827-1834. https://doi.org/10.1007/ s11695-012-0726-9

Luca P, Nicolas C, Marina V, Sarah B \& Andrea L (2021). Where are my patients? lost and found in bariatric surgery. Obes Surg 31(5):19791985. https://doi.org/10.1007/s11695-02005186-9

Lujan J, Tuero C, Landecho MF, Moncada R, Cienfuegos JA, Rotellar F, Silva C, Lapuente F, Martínez P, Frühbeck G \& Valenti V (2020). Impact of routine and long-term follow-up on weight loss after bariatric surgery. Obes Surg 30(11): 4293-4299. https://doi.org/10.1007/ s 11695-020-04788-7

Maïmoun L, Lefebvre P, Aouinti S, Picot MC, Mariano-Goulart D \& Nocca D (2019). Acute and longer-term body composition changes after bariatric surgery. Surg Obes Relat Dis 15(11):1965-1973. https://doi.org/10.1016/j. soard.2019.07.006

Masnyj SV, Shea BJ \& Khaitan L (2020). Predictors of success in bariatric surgery. Curr Cardiovasc Risk Rep 14(3):3. https://doi.org/10.1007/ s12170-020-0637-6

Mittal T, Ahuja A, Dey A, Malik VK, Mustafa Sheikh MT, Bansal NK \& Kanuri H (2021). Safety and efficacy of laparoscopic sleeve gastrectomy in patients with portal hypertension with liver function of Childs A. Surgical Endoscopy https://doi.org/10.1007/s00464-021-08587-8

MOHAP (2019). New International Accreditation Received by Al Qassimi Hospital's Bariatric Surgery Center from the Surgical Evaluation Corporation (SRC)-Ministry of Health and Prevention-UAE. https://www.mohap.gov. ae/en/MediaCenter/News / Pages/2217.aspx [Retrieved July 9 2021].

Nimeri A, AlHadad M \& Khoursheed M (2017). The peri-operative bariatric surgery care in the Middle East Region. Obes Surg 27(6):15431547. https://doi.org/10.1007/s11695-0162503-7
Nuijten MAH, Monpellier VM, Eijsvogels TMH, Janssen IMC, Hazebroek EJ \& Hopman MTE (2020). Rate and determinants of excessive fatfree mass loss after bariatric surgery. Obes Surg 30(8):3119-3126. https://doi.org/10.1007/ s1 1695-020-04654-6

Palacio A, Quintiliano D, Lira I, Navarro P, Orellana V, Reyes A \& Henriquez D (2019). Changes in body composition in patients following bariatric surgery: Gastric bypass and sleeve gastrectomy. Nutr Hosp 36(2):334-339. https:/ / doi.org/10.20960/nh.2255

Pareek M, Schauer PR, Kaplan LM, Leiter LA, Rubino F \& Bhatt DL (2018). Metabolic Surgery. J Am Coll Cardiol 71(6): 670-687. https://doi. org/10.1016/j.jacc.2017.12.014

Ramos A, Kow L, Welbourn R, Dixon J, Kinsman R \& Walton P (2019). $5^{\text {th }}$ IFSO Global Registry Report. IFSO \& Dendrite Clinical Systems. https: / / www.ifso.com/pdf/5th-ifso-globalregistry-report-september-2019.pdf [Retrieved July 9 2021].

Ruban A, Stoenchev K, Ashrafian H \& Teare J (2019). Current treatments for obesity. Clin Med 19(3):205-212. https://doi.org/10.7861/ clinmedicine. 19-3-205

Rutledge R, Kular KS, Manchanda N, Bhandari M \& Goel R (2017). A comparison of the outcomes revision of the roux-en-y and mini-gastric bypass: Hard vs. Easy. Laparosc Endosc Surg Sci 24(2):58-62. https://doi.org/10.14744/ less. 2017.69188

Schwoerer A, Kasten K, Celio A, Pories W \& Spaniolas $\mathrm{K}$ (2017). The effect of close postoperative followup on co-morbidity improvement after bariatric surgery. Surg Obes Relat Dis 13(8):1347-1352. https://doi.org/10.1016/j.soard.2017.03.024

Sulaiman N, Elbadawi S, Hussein A, Salah Abusnana, Madani A, Mairghani M, Alawadi F, Sulaiman A, Zimmet P, Huse O, Shaw J \& Peeters A (2017). Prevalence of overweight and obesity in United Arab Emirates Expatriates: The UAE National Diabetes and Lifestyle Study. Diabetol Metab Syn 9:88. https:/ / doi.org/ 10.1186/s 13098-017-0287-0

Tu YF, Pan YH, Han JF, Pan JM, Zhang P, Jia WP, Bao YQ \& Yu HY(2021). A total weight loss of $25 \%$ shows better predictivity in evaluating the efficiency of bariatric surgery. Int $J$ Obes 45(2):396-403. https://doi.org/10.1038/ s41366-020-00690-5 
Unamuno X, Portincasa P \& Frühbeck G (2019). A paradigm shift in bariatric surgery outcome evaluation? Lancet Diabetes \& Endocrinol 7(10):743-745. https://doi.org/10.1016/ S2213-8587(19)30248-7

World Health Organization/Regional Office for The Eastern Mediterranean (2018). WHO EMRO | Obesity | Health topics.. From http:// www.emro.who.int/health-topics /obesity/ [Retrieved July 9 2021]
Zalesin KC, Franklin BA, Lillystone MA, Shamoun T, Krause KR, Chengelis DL, Mucci SJ, Shaheen KW \& McCullough PA (2010). Differential loss of fat and lean mass in the morbidly obese after bariatric surgery. Metab Syndr Relat Disord 8(1):15-20. doi: 10.1089/met.2009.0012. PMID: 19929598. 